ARTICLE

DOI: $10.1038 /$ s41467-018-03245-5

\title{
Cell-based glycan arrays for probing glycan-glycan binding protein interactions
}

Jennie Grace Briard ${ }^{1}$, Hao Jiang ${ }^{2}$, Kelley W. Moremen ${ }^{3}$, Matthew Scott Macauley ${ }^{1,4}$ \& Peng Wu (i) ${ }^{1}$

Glycan microarrays provide a high-throughput means of profiling the interactions of glycanbinding proteins with their ligands. However, the construction of current glycan microarray platforms is time consuming and expensive. Here, we report a fast and cost-effective method for the assembly of cell-based glycan arrays to probe glycan-glycan-binding protein interactions directly on the cell surface. Chinese hamster ovary cell mutants with a narrow and relatively homogeneous repertoire of glycoforms serve as the foundation platforms to develop these arrays. Using recombinant glycosyltransferases, sialic acid, fucose, and analogs thereof are installed on cell-surface glycans to form cell-based arrays displaying diverse glycan epitopes that can be probed with glycan-binding proteins by flow cytometry. Using this platform, high-affinity glycan ligands are discovered for Siglec-15-a sialic acid-binding lectin involved in osteoclast differentiation. Incubating human osteoprogenitor cells with cells displaying a high-affinity Siglec-15 ligand impairs osteoclast differentiation, demonstrating the utility of this cell-based glycan array technology.

\footnotetext{
${ }^{1}$ Department of Molecular Medicine, The Scripps Research Institute, La Jolla, CA 92037, USA. ${ }^{2}$ Key Laboratory of Marine Drugs, Ministry of Education and Qingdao National Laboratory for Marine Science \& Technology and Shandong Provincial Key Laboratory of Glycoscience \& Glycoengineering, School of Medicine and Pharmacy, Ocean University of China, 266003 Qingdao, China. ${ }^{3}$ Complex Carbohydrate Research Center and the Department of Biochemistry and Molecular Biology, University of Georgia, Athens, GA 30602, USA. ${ }^{4}$ Present address: Department of Chemistry, University of Alberta, Edmonton, AB T6G 2G2, Canada. Correspondence and requests for materials should be addressed to M.S.M. (email: macauley@ualberta.ca) or to P.W. (email: pengwu@scripps.edu)
} 
G lycans decorate the cell surface of both eukaryotes and prokaryotes, and in mammalian cells are involved in a variety of physiological processes, including angiogenesis, fertilization, stem cell development, and neuronal development $t^{1-3}$. Changes in glycosylation patterns have also been shown to mark the onset of cancer and inflammation ${ }^{2,3}$. In many cases, glycans execute these cellular functions by interacting with glycanbinding proteins (GBPs). Therefore, there is enormous interest in understanding the structural basis of these interactions for the dissection of the mechanisms of glycan-mediated biological processes and for the development of new therapeutic agents to treat glycan-regulated disease. Unfortunately, it is challenging to probe glycan-GBP interactions in vivo because glycosylation is a post-translational modification not under direct genetic control. The dynamic process of glycosylation orchestrated by glycosylation enzymes results in heterogeneous glycoconjugates found on the cell surface and on secreted proteins ${ }^{3}$.

Glycan microarrays were developed in response to the critical need for high-throughput methods to identify GBP interactions ${ }^{4,5}$. As highlighted in Transforming Glycoscience (section 5.1.1), these microarrays have been extensively employed to interrogate binding specificities of a diverse range of GBPs, determine dissociation constants, dissect binding energies, and assess multivalent and hetero-ligand binding ${ }^{6}$. Currently, most glycan arrays are constructed by coupling a chemically defined glycan to a solid support, such as a glass slide ${ }^{4,5}$. Such homogeneous glycans and derivatives are either synthesized ${ }^{4}$ or purified from natural sources by multi-dimensional chromatography ${ }^{7}$. Several noteworthy drawbacks are associated with the current platforms. First, obtaining samples of pure, wellcharacterized oligosaccharides for the assembly of glycan arrays by chemical or chromatography-based purification is time consuming and can only be performed by a specialist. As such, glycosyltransferases are often employed in combination with chemical synthesis to facilitate the production of complex oligosaccharides ${ }^{8}$. However, only limited numbers of glycosyltransferases are present in carbohydrate chemists' toolbox. Therefore, many glycosidic linkages cannot be assembled in a straightforward manner. The second drawback is that the current glycan microarrays do not fully recapitulate the natural cellsurface environment on which glycans are presented. Indeed, Wong and co-workers have shown that the poor sensitivity of the conventional microarrays arises from their surface-generated pseudo-multivalent display9. To better mimic the natural multivalent presentation, several groups have developed creative strategies by attaching synthetic glycans to $\operatorname{protein}^{10}$ or polymer scaffolds $^{11}$. These approaches, however, also rely on the lengthy synthesis of complex glycans.

Here, we describe a method to chemoenzymatically install monosaccharides and their analogs directly on the cell surface to create in-solution, cell-based arrays displaying chemically defined peripheral glycan epitopes. The lectin-resistant Chinese hamster ovary $(\mathrm{CHO})$ cell mutant Lec2 that expresses a narrow and relatively homogenous repertoire of glycoforms is employed as the foundation platform. With the conserved core glycan structures already expressed on the cell surface, the lengthy synthesis required to build complex carbohydrates is avoided. Using a handful of glycosyltransferases compatible with cell-surface glycosylation, sialic acid, fucose, and their analogs are introduced to these cells' peripheral glycans linkage specifically to form cellbased arrays displaying diverse glycan epitopes. We demonstrate the utility of these cell-based arrays to interrogate GBP specificities and ligand tolerance directly on the cell surface. This method is applied to high throughput screening for the identification of selective and high-affinity ligands of Siglecs, a family of sialic acid-binding immunoglobulin-type lectins that are differentially expressed primarily on immune cells. Using this approach, a high-affinity glycan ligand for Siglec-15 is discovered that can be used to modulate the differentiation of osteoclasts.

\section{Results}

Design and validation of cell-based glycan array strategy. As proof-of-principle, we used the $\mathrm{CHO}$ glycosylation mutant Lec2 cells ${ }^{12}$ to construct in-solution, cell-based glycan arrays displaying defined periphery glycans (Fig. 1a). Lec2 cells have an inactive CMP-sialic acid Golgi transporter. As a consequence, no sialylation occur without the donor substrate CMP sialic acid avaliable in the Golgi. In addition, there are no active $\alpha 1-2$, $\alpha 1-3$, and $\alpha 1-4$ fucosyltransferases (FTs) and, therefore, their cell-surface N-glycans terminate with $N$-acetyllactosamine (LacNAc, Gal $\beta 1$ 4GlcNAc) (Fig. 2c) ${ }^{12}$. Lacking terminal fucose or sialic acid (Fig. 1a) makes Lec2 cells a rich source of acceptor substrates for FTs and sialyltransferases (STs). Accordingly, Helicobacter pylori a1-3FT ${ }^{13-15}$, human a2-3ST (ST3Gal4) $)^{16}$, and rat a2-6ST (ST6Gal1) $^{16-18}$ were employed to install fucose or sialic acid onto the cell surface in a linkage-specific manner. The addition of a1-3-linked fucose leads to the formation of Lewis X (Gal $\beta 1-4$ (Fuca1-3)GlcNAc, Le ${ }^{\mathrm{X}}$ ) (Fig. 1b). The addition of $\alpha 2-6-$ or $\alpha 2-3$ linked sialic acid leads to the formation of Siaa2-6Gal $\beta 1$ 4GlcNAc or Siaa2-3Galß1-4GlcNAc, respectively (Fig. 1d).

The cells displaying Le ${ }^{\mathrm{X}}$ were constructed by treating Lec2 cells with GDP-fucose (GDP-Fuc) (1) $(500 \mu \mathrm{M})$ and $\alpha 1-3 \mathrm{FT}$ in Hanks buffered salt solution (HBSS) for $10 \mathrm{~min}$ at $37^{\circ} \mathrm{C}$ (Fig. 2a). Lec8 $\mathrm{CHO}$ cells that do not express LacNAc due to a mutation in the UDP-Gal Golgi transporter were used as a negative control ${ }^{19}$. The modified cells were then probed with fucoside binding proteins or antibodies, including anti-stage-specific embryonic antigen-1 (anti-SSEA-1 or anti-Le ${ }^{\mathrm{X}}$ ), Aleuria aurantia lectin (AAL), and Lotus tetragonolobus lectin (Lotus A) as FITC conjugates ${ }^{20}$. Flow cytometry reveals that anti-SSEA-1, AAL, and Lotus A all exhibited strong binding to cells displaying the $\mathrm{Le}^{\mathrm{X}}$ epitope (Fig. 2b). In contrast, only background binding was observed with non-fucosylated Lec 2 cells and control Lec8 cells.

The cells displaying Siaa2-3Gal $\beta 1-4 \mathrm{GlcNAc}$ and Siaa2-6Gal $\beta 1$ $4 \mathrm{GlcNAc}$ were constructed by treatment of Lec2 cells with CMPNeu5Ac (6) (Fig. 2a) and ST3Gal4 or ST6Gal1, respectively, for 1 $\mathrm{h}$ at $37^{\circ} \mathrm{C}$. Again, Lec8 CHO cells were used as a negative control. These two cells were first probed with FITC-conjugated Erythrina cristagalli lectin (ECL) that is specific for terminal galactose and $\mathrm{N}$-acetylgalactosamine and as such, bound strongly to unmodified Lec2 CHO cells (Fig. 2c) ${ }^{21}$. Consistent with the specificity of ECL, the cells with installed sialic acid resulted in reduced binding (Fig. 2c). The cells were then probed with FITC-conjugated sialoside binding proteins, Sambucus nigra (SNA-I) and Maackia amurensis lectin (MAL-II), specific for a2-6- and a2-3-linked sialic acids, respectively ${ }^{20}$. SNA-I strongly bound to cells displaying a2-6-linked sialic acid, whereas no binding was observed for cells with a2-3-linked sialic acid (Fig. 2c). For MAL-II, the opposite was observed (Fig. 2c). In these experiments, as little as $1 \mu \mathrm{g} / \mathrm{mL}$ of GBPs was used. Together, these results provide strong evidence that our approach for installing monosaccharides to construct ligands for probing their interactions with GBPs on cell surfaces is specific and sensitive.

Construct glycan arrays displaying unnatural epitopes. Screening glycan libraries with unnatural substituents serves as a powerful means to probe the binding pocket of a GBP especially when there is limited structural information available. To apply cell-based glycan arrays to profile the substrate tolerance of GBPs, we constructed three arrays displaying $\mathrm{Le}^{\mathrm{X}}$, Siaa2-3Gal $\beta 1$ $4 \mathrm{GlcNAc}$, Siaa2-6Gal $\beta 1-4 \mathrm{GlcNAc}$ and their structurally related 
a
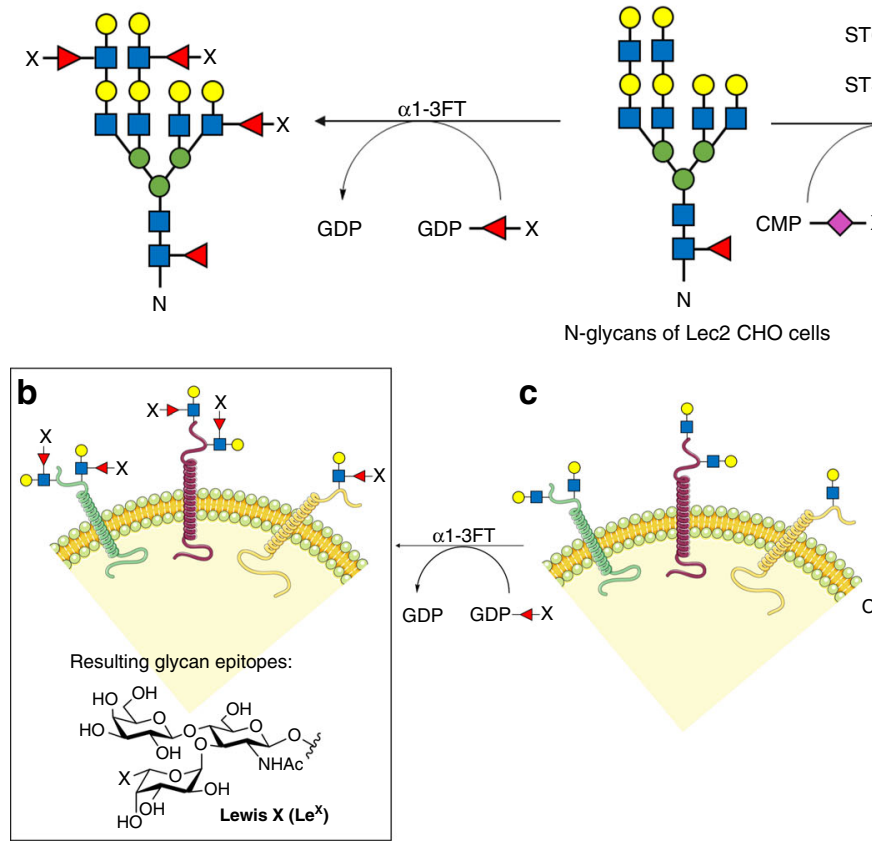

N-glycans of Lec2 $\mathrm{CHO}$ cells
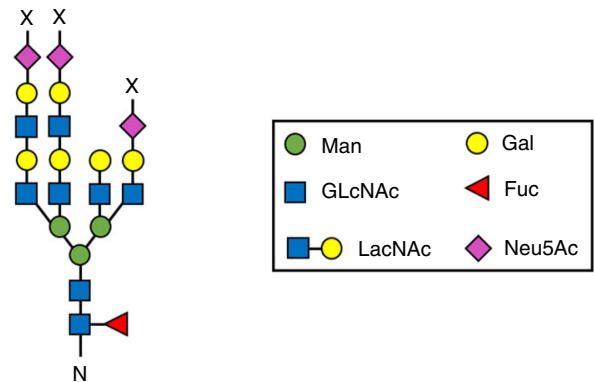

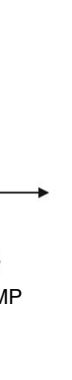

N

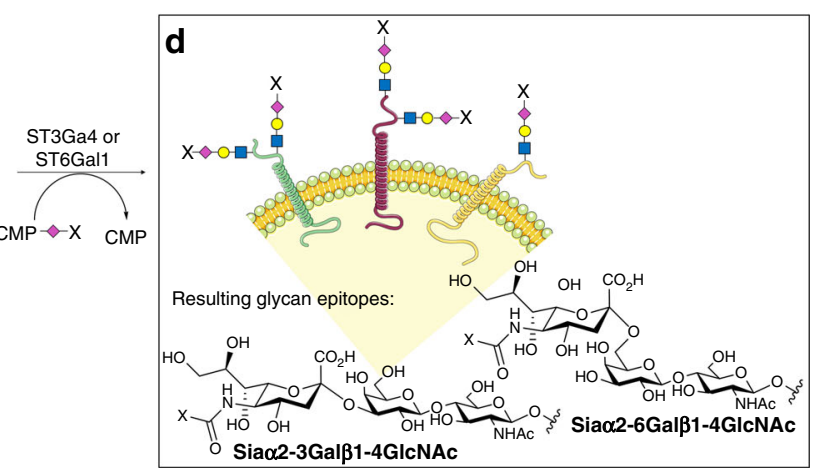

Fig. 1 The workflow for constructing cell-based glycan arrays. a Distinct glycan epitopes are installed onto N-glycans of Lec2 CHO cells via sialyltransferase-mediated cell-surface in situ sialylation using the CMP-Neu5Ac donor (or its analogs) or fucosyltransferase-mediated cell-surface in situ fucosylation using the GDP-Fuc donor (or its analogs). b Cell-based glycan arrays displaying Le ${ }^{X}$ or its derivatives. c N-glycans of Lec2 CHO cells. d Cellbased glycan arrays displaying $\alpha 2-3-$ or $\alpha 2-6$-linked sialosides, or their derivatives. $\mathrm{X}$ represents unnatural substituents introduced at fucose $\mathrm{C}-5$ or NeuNAc acetyl position

derivatives using Lec2 $\mathrm{CHO}$ cells as the starting platform. The array displaying $\mathrm{Le}^{\mathrm{X}}$ and its derivatives was constructed using $500 \mu \mathrm{M}$ GDP-Fuc (1) or GDP-Fuc analogs (2-5) (Fig. 3a). Our previous studies revealed that while a1-3FT is highly specific for acceptor glycans possessing LacNAc, it has relaxed donor substrate specificity and is able to accept GDP-Fuc donors with a wide variety of functional groups at the C5 position of fucose ${ }^{15}$. Kinetic analysis of the enzymatic transformation showed only minor differences $(2.8,8.4,6.2,24 \mu \mathrm{M}$ for 2-5 vs. $16 \mu \mathrm{M}$ for GDPFuc (1)) in $K_{\mathrm{m}}$ values for GDP-Fuc donors with functional groups at C5. Furthermore, there was no appreciable influence on the $V_{\max }$ (Supplementary Fig. 1). Therefore, we chose $500 \mu \mathrm{M}$ as the concentration for all donor substrates for surface fucosylation, which was well above the saturated substrate concentration.

The arrays displaying Siaa2-3Gal $\beta 1-4 \mathrm{GlcNAc}$, Siaa2-6Gal $\beta 1$ $4 \mathrm{GlcNAc}$ and their derivatives were constructed using CMPNeu5Ac (6) or CMP-Neu5Ac analogs (7-9) (Fig. 3b). Upon sialylation by ST3Gal4 and ST6Gal1, Lec2 CHO cells showed a reduction in ECL binding indicating donor transfer regardless of the sialic analog used (Supplementary Fig. 2). This observation is consistent with an earlier discovery showing that ST6Gall was promiscuous for C5-modified CMP-Neu5Ac analogs and could even tolerate large C5 moieties like biotin ${ }^{22,23}$.

The array displaying $\mathrm{Le}^{\mathrm{X}}$ and its derivatives was probed with fucoside binding proteins, SSEA-1, AAL, and Lotus A. The arrays displaying Siaa2-3Gal $\beta 1-4$ GlcNAc, Siaa2-6Gal $\beta 1-4 G l c N A c$ and their derivatives were probed with SNA-I and MAL-II. Interestingly, we discovered that this panel of GBPs exhibited distinct binding tolerances to unnatural glycan derivatives. Anti-SSEA-1, also known as anti-Le ${ }^{\mathrm{X}}$ antibody, was able to tolerate an alkyne (2) and a hydroxyl group (4) at the C5 position of fucose, but not an azide (3) or methoxy (5) functional group, which abrogated interaction (Fig. 3c). All fucose analogs assessed resulted in a reduction in AAL binding but did not entirely abolish this interaction. Lotus A was very sensitive to $\mathrm{C} 5$ modification and the interaction was completely blocked by any modification to the C5 position of fucose. On the other hand, SNA-I and MAL-II were not sensitive to sialic acid modifications at $\mathrm{C} 5$, but demonstrated linkage-specificity (Fig. 3d). SNA-I bound to all sialic acid analogs when attached in an a2-6-linkage. Alternatively, MAL-II tolerated all analogs attached in an a2-3-linkage.

Screen high-affinity Siglec-15 ligands. Having demonstrated that our cell-based glycan array was capable of detecting GBP binding specificity and substrate tolerance, we next assessed the applicability of this method to identify high-affinity Siglec ligands. Siglecs comprise a family of 15 members of sialic acid-binding receptors that are differentially expressed on immune cells ${ }^{24-26}$. Because of the restricted expression of Siglecs to one or a few immune cell types, Siglecs are attractive targets for cell-directed therapies in immune-cell-mediated diseases ${ }^{26-29}$. Furthermore, Siglecs are endocytic receptors allowing efficient uptake of therapeutic agents conjugated to an antibody or glycan ligand ${ }^{30-33}$. High-affinity and highly selective glycan ligands represent attractive alternatives to antibody-based therapeutics with several notable advantages. Notably, nanoparticles bearing sialoside ligands for delivery of therapeutic cargo have shown promise for targeting Siglecs in vivo ${ }^{34}$. To date, high-affinity and selective glycan ligands have been discovered for approximately half of human Siglecs using conventional glycan microarray technology $^{35-38}$.

We sought to determine the applicability of our cell-based array technique to discover high-affinity and selective glycan ligands for Siglecs. One Siglec of interest where high-affinity and selective ligands have not been discovered is Siglec-15. Siglec-15 


\begin{tabular}{|c|c|c|c|}
\hline 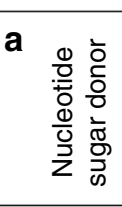 & $\begin{array}{l}\mathrm{OTOH} \\
\mathrm{HO} \\
\text { GDP-Fuc (1) }\end{array}$ & CMP-Sialic acid (6) & CMP-Sialic acid (6) \\
\hline$\stackrel{\text { 保 }}{ }$ & $\alpha 1-3 \mathrm{FT}$ & ST6Gal1 & ST3Gal4 \\
\hline
\end{tabular}
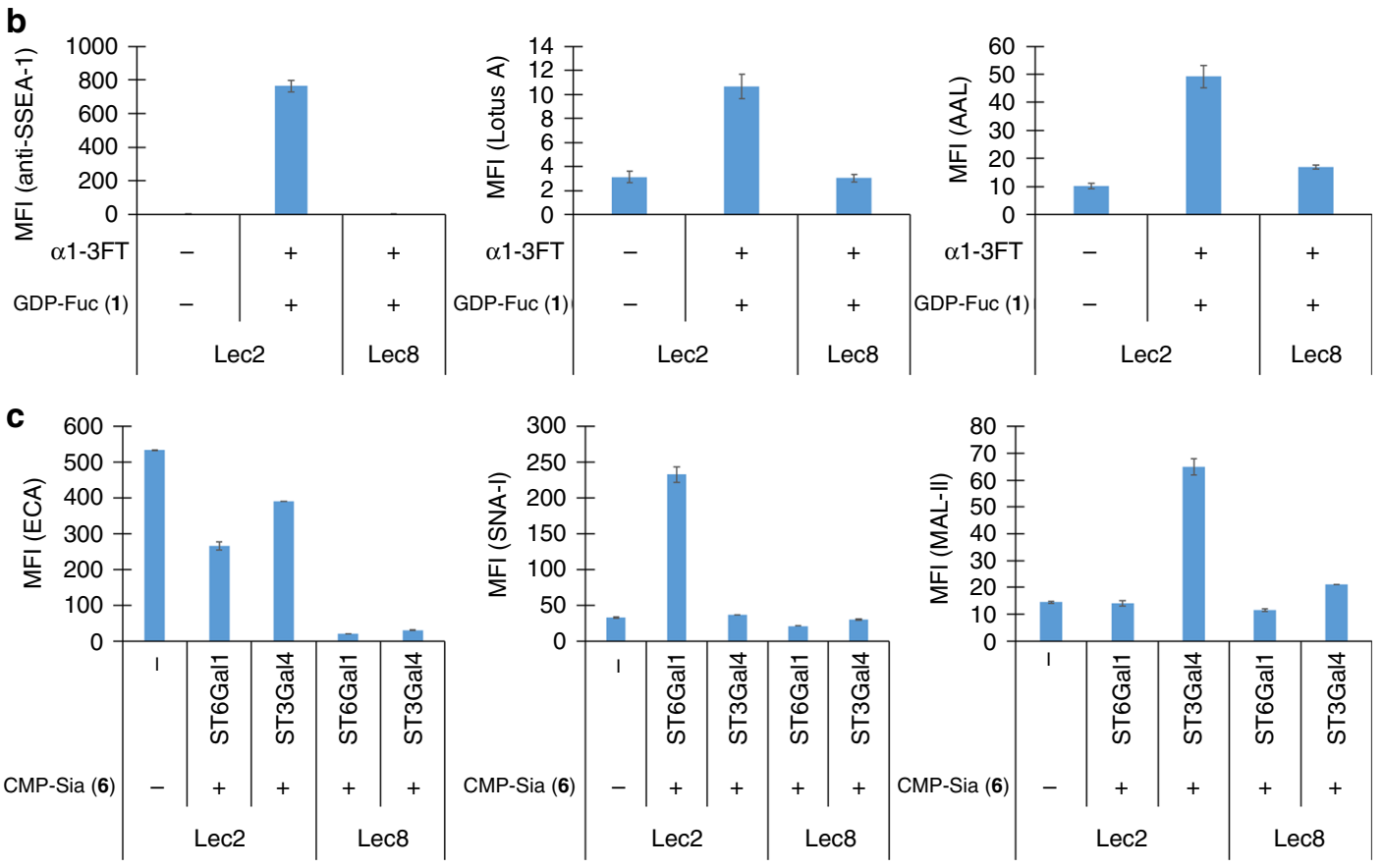

Fig. 2 Validate the specificity of in situ glycosylation strategy. a Nucleotide sugar donors and glycosyltransferases used to form three glycan epitopes (Le ${ }^{X}$,

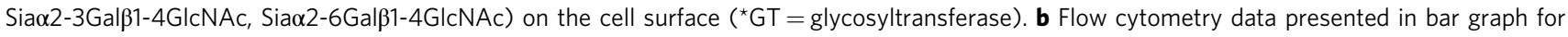
validating cell-surface in situ fucosylation. $\mathrm{CHO}$ cells were modified via in situ fucosylation using the donor GDP-Fuc (1) and $\alpha 1-3 \mathrm{FT}$. The modified cells were probed with a fluorescently labeled lectin or antibody and analyzed by flow cytometry $(n=3$, error bars are reported as the standard error of the mean (SEM)). c Flow cytometry data presented in bar graph for validating in situ sialylation. CHO cells were modified via in situ sialylation using the donor CMP-Sia (6) and the sialyltransferase ST6Gal1 or ST3Gal4. The modified cells were probed with a fluorescently labeled lectin and analyzed by flow cytometry $(n=3$, error bars are reported as SEM)

becomes expressed within days of RANKL (receptor activator of nuclear factor $\kappa \beta$ ligand)-induced differentiation of osteoclast precursors into osteoclasts ${ }^{39-43}$. Studies have demonstrated that Siglec-15 positively regulates osteoclast differentiation and, consistent with this finding, loss of Siglec-15 results in impaired osteoclast differentiation and osteopetrosis in Siglec-15-deficient mice ${ }^{40}$. Therefore, Siglec-15 plays a critical role in bone remodeling and is a considerable potential therapeutic target for osteoporosis.

From what is known, Siglec-15 prefers a2-6-linked sialic acid residues in native ligands ${ }^{43}$. As several high-affinity Siglec ligands have been developed with functional modifications at the C5 position of Neu5Ac, we focused on this position on sialic acid to create chemical diversity ${ }^{35-38}$. To do so, we employed ST6Gall to install an a2-6-linked sialic acid bearing a bioorthogonal alkyne tag at C5 onto the cell surface of Lec2 cells (Fig. 4a). The alkyne tagged cells were then derivatized by reaction with a 40 -member azide library (Supplementary Table 1) via biocompatible $\mathrm{Cu}(\mathrm{I})$ catalyzed azide-alkyne cycloaddition (CuAAC) (Fig. $4 \mathrm{a})^{44}$ to form a cell-based glycan array. The library was chosen to contain structures with a variety of different functional groups to cover a range of chemical space. The resulting cell-based glycan array was probed with a recombinant Siglec-15-Fc chimera pre-complexed to APC-conjugated anti-human IgG1. From this screen, several high-affinity glycan ligands for Siglec-15 were discovered (Fig. 4b). Three structures (derived from azides A13, A31, and A37) resulted in an increase in Siglec-15-Fc binding greater than 15fold compared to Lec2 $\mathrm{CHO}$ cells sialylated with native sialic acid (6).

To determine if sialic acid derivatives functionalized with A13 and A31 still serve as high-affinity ligands for Siglec-15 when displayed on the cell surface in different linkages, CMP-SiaA13 and CMP-SiaA31 (10 and 11; Fig. 4c) were synthesized and used as the donor substrates for cell-surface in situ sialylation mediated by three sialyltransfereases, ST6Gal1, ST3Gal4, and ST3Gal1, followed by Siglec-15-Fc binding. First, the ability of each enzyme to tolerate the alkyne-tagged CMP-SiaPoc (7) and CMP-Neu5Ac analogs with larger functional groups (e.g. biotin) at C5 was verified by the two-step (transfer CMP-SiaPoc in step 1 and react with biotin-azide in step 2) and one-step (directly transfer a C5 biotin functionalized Neu5Ac analog) labeling, respectively, and followed by detection with streptavidin-APC (Supplementary Fig. 3). The one-step procedure was found to be more efficient than the two-step method for all sialyltransferases, consistent with previous reports ${ }^{23}$. Lec2 $\mathrm{CHO}$ cells were then subjected to the one-step labeling with 10 and 11 by ST6Gal1, ST3Gal4, or ST3Gal1. ST6Gal1 and ST3Gal4 install CMP-Neu5Ac analogs on terminal LacNAc residues of $\mathrm{N}$-glycans resulting in a display of 


\begin{tabular}{|c|c|c|c|c|c|}
\hline 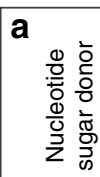 & $\begin{array}{l}\mathrm{O} \mathrm{TOH}^{\mathrm{OGDP}} \\
\mathrm{OH} \\
\text { GDP-Fuc (1) }\end{array}$ & $=\sqrt{\mathrm{OH}_{2}^{\mathrm{OH}}} \mathrm{TH}^{\mathrm{OGDP}}$ & $\underbrace{\mathrm{N}}_{\mathrm{OH}} \mathrm{ZOH}_{3} \mathrm{OGDP}$ & $\sqrt{\mathrm{OH}}_{4}^{\mathrm{O}} \mathrm{TOH}^{\mathrm{OGDP}}$ & $\mathrm{HO}_{5}$ \\
\hline$\stackrel{*}{*}$ & \multicolumn{5}{|c|}{$\alpha 1-3 \mathrm{FT}$} \\
\hline 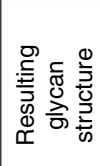 & 管 & (OH & (OH & (N) & 管 \\
\hline
\end{tabular}

\begin{tabular}{|c|c|c|c|c|}
\hline 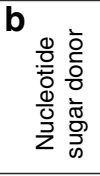 & (6) & $=\prod_{7}^{\mathrm{HO}}$ & $\left.\right|_{0} ^{\mathrm{HO}}$ & 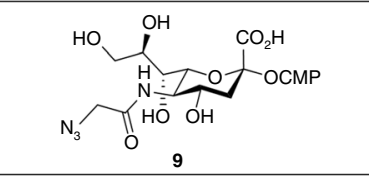 \\
\hline 感 & \multicolumn{4}{|c|}{ ST6Gal1/ST3Gal4 } \\
\hline 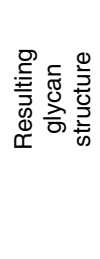 & (n) & (n) & (NHAC & (n) \\
\hline
\end{tabular}

C
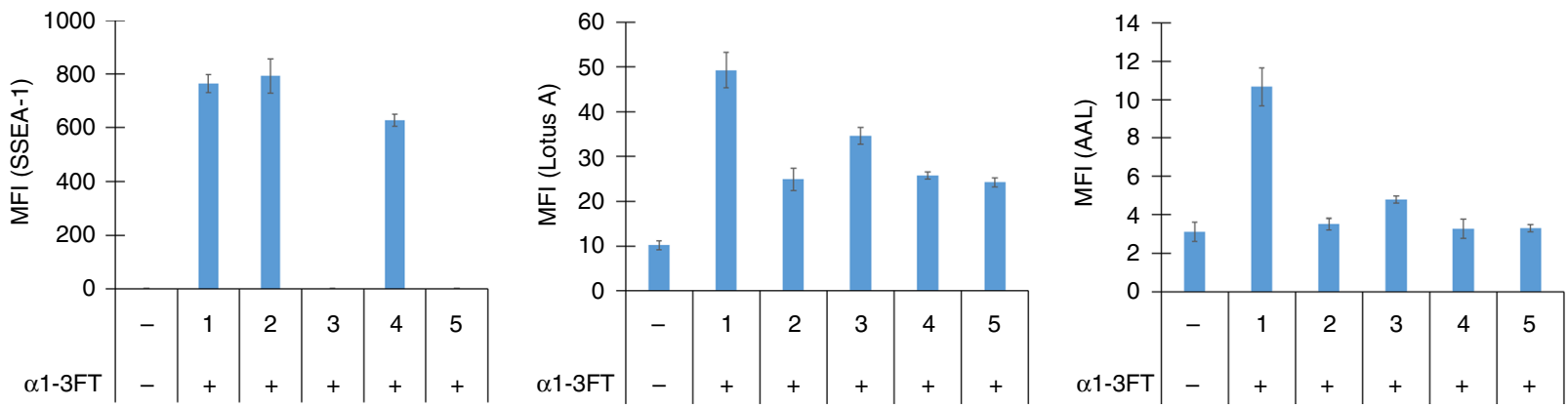

d
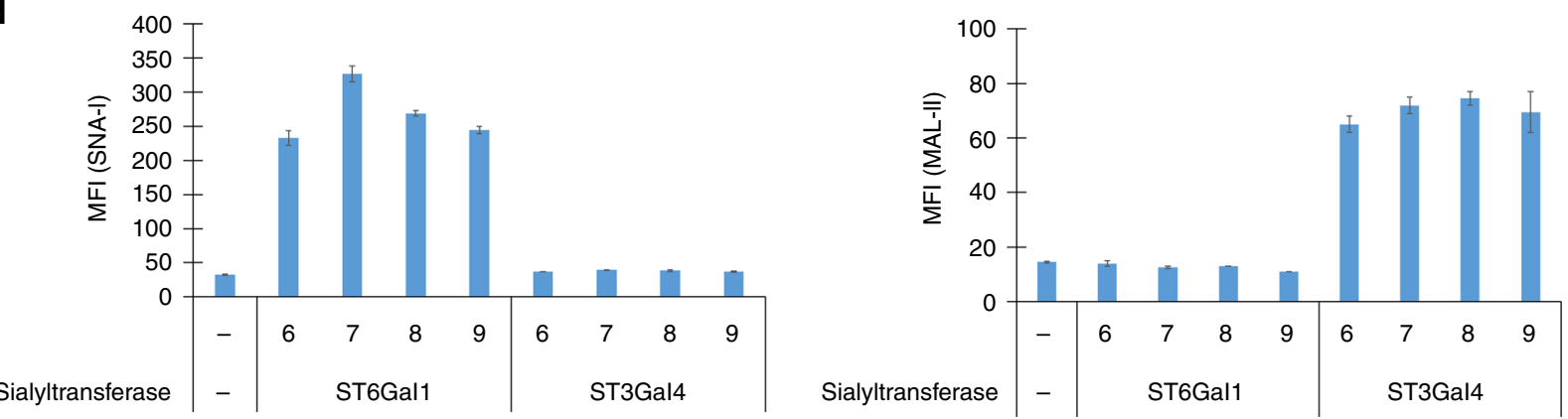

Fig. 3 Use cell-based glycan arrays to profile GBP substrate scope. a GDP-Fuc(1), GDP-Fuc analogs (2-5) and resulting glycan structures after in situ cell-surface fucosylation. b CMP-sialic acid (6), CMP-sialic acid analogs (7-9) and resulting glycan structures after cell-surface sialylation. c Flow cytometry data presented in bar graph for profiling substrate tolerance of fucose-binding lectins and antibodies. $\mathrm{CHO}$ cells were modified via in situ fucosylation using $\alpha 1-3 F T$ and the donor substrate GDP-Fuc or its analogs. The modified cells were probed with a fluorescently labeled lectin or antibody and analyzed by flow cytometry $(n=3$, error bars are reported as SEM). $\mathbf{d}$ Flow cytometry data presented in bar graph for profiling substrate tolerance of sialic acid-binding lectins. CHO cells were modified via in situ sialylation using the sialyltransferase ST6Gal1 or ST3Gal4 in the presence of the donor substrate CMP-Sia or its analogs. The modified cells were probed with a fluorescently labeled lectin and analyzed by flow cytometry $(n=3$, error bars are reported as SEM)

a2-6- or $\mathbf{\alpha 2 - 3 - 1 0}$ or -11 sialosides, respectively, whereas ST3Gal1 is responsible for transferring Neu5Ac analogs to the galactose of $\mathrm{Gal} \beta(1-3) \mathrm{GalNAc}$ on $\mathrm{O}$-glycans to form a2-3-linked sialosides (Fig. 4c) ${ }^{13,37,45}$. Siglec-15 bound to both $\boldsymbol{\alpha} 2-\mathbf{6}-\mathbf{1 0}$ and $\boldsymbol{\alpha 2 - 3 - 1 0}$ on N-glycans with similar affinity (Fig. 4d). By contrast, Siglec-15 preferred sialoside $\boldsymbol{\alpha} 2-\mathbf{6 - 1 1}$ on N-glycans. Interestingly, Siglec-15 did not bind to either $\mathbf{1 0}$ or $\mathbf{1 1}$ derived sialosides when displayed in a2-3-linkages on O-glycans.

Many high-affinity glycan ligands for Siglecs lack the necessary specificity required for downstream applications ${ }^{35}$. To examine 
the specificity of the Siglec-15 glycan ligands discovered here, we analyzed the binding of a panel of human Siglec-Fc chimeras to Lec2 CHO cells displaying $\boldsymbol{\alpha 2 - 6 - 1 0}$ or $\boldsymbol{\alpha 2 - 6 - 1 1}$ (Fig. 4e). Both $\boldsymbol{\alpha 2 -}$ 6-10 and a2-6-11 were found to be high-affinity ligands for Siglec-2, with a 123- and 81-fold increase in Siglec-2-Fc binding compared to the negative control, respectively. Cells displaying a2-6-10 also showed weak binding to Siglec-10-Fc. Subsequently, we screened the same panel of Siglecs using cells displaying $\mathbf{\alpha 2 - 3 -}$ 10 and $\boldsymbol{\alpha}$ 2-3-11 (Fig. 4f). Interestingly, both $\boldsymbol{\alpha} 2-3-10$ and $\boldsymbol{\alpha} 2-3-11$ showed dramatically increased specificity for Siglec-15-the

a
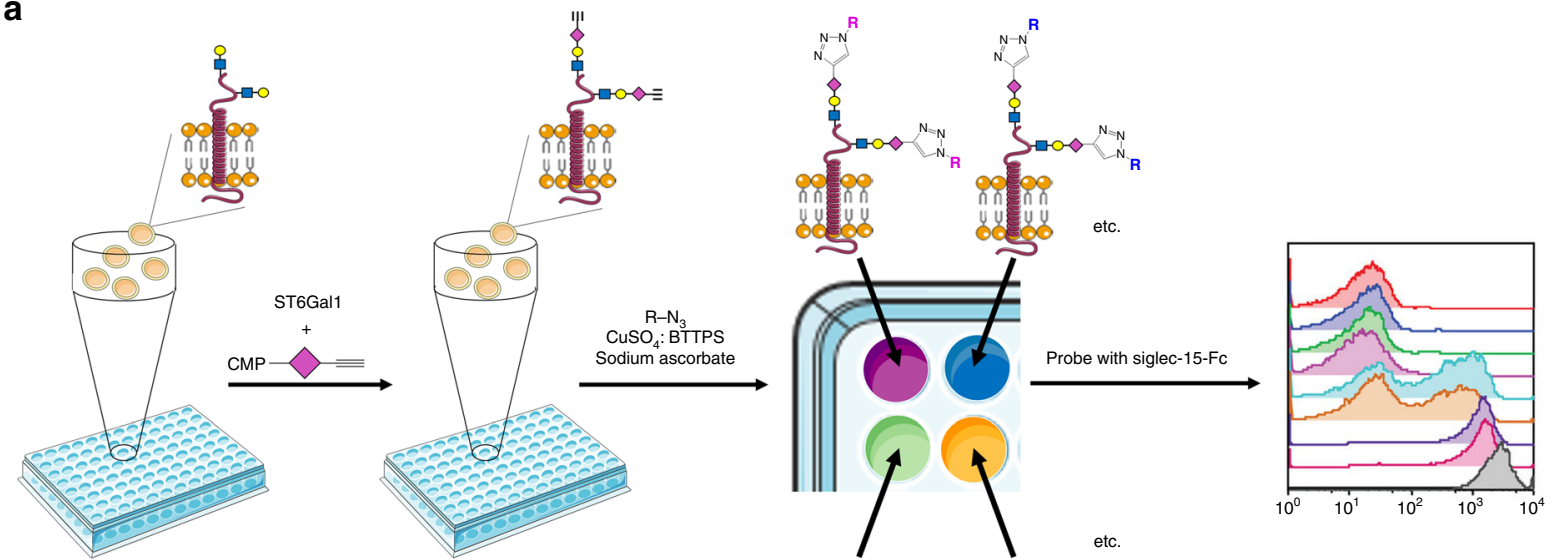

b

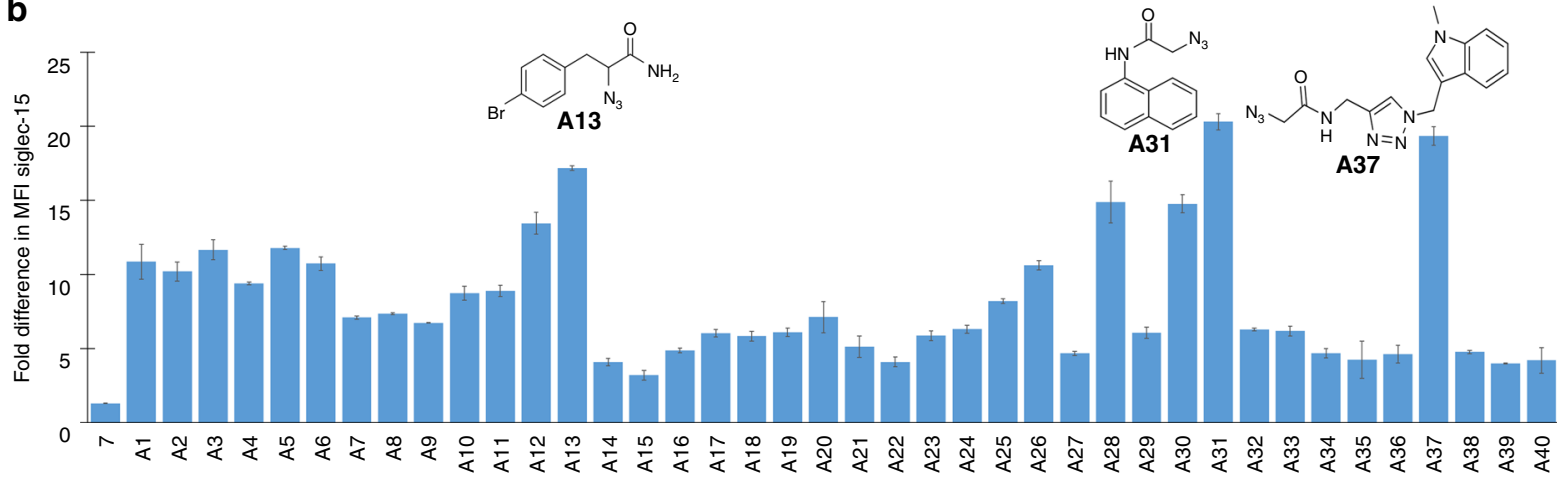

C
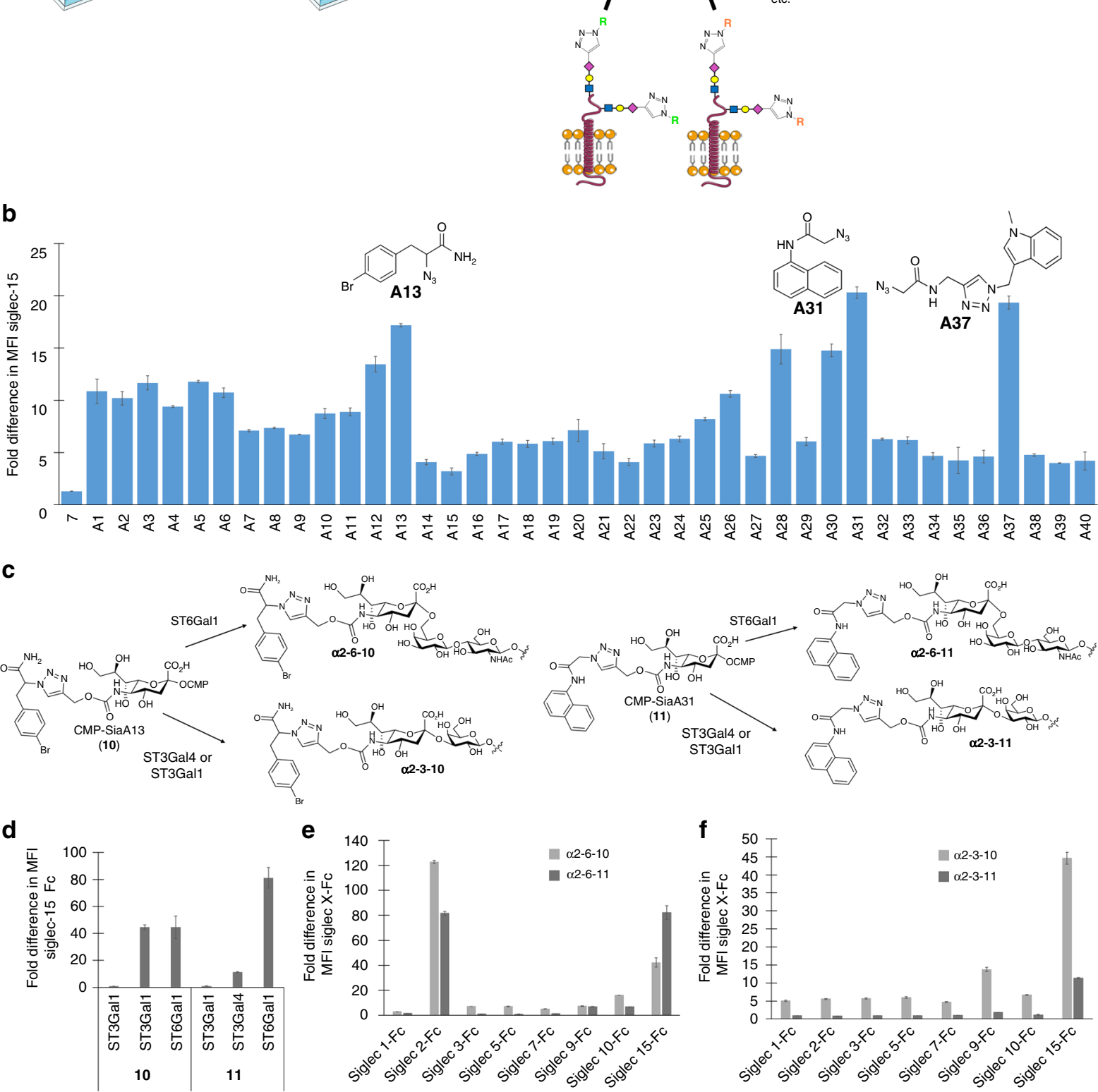
interaction with Siglec- 2 was completely abolished. Although cells displaying a2-3-10 still exhibited weak binding to Siglec-9, cells displaying a2-3-11 showed remarkable specificity for Siglec-15 with negligible binding to all other Siglec-Fc chimeras assessed.

High-affinity Siglec-15 ligands engage in cell-cell interactions. With the identification that $\mathbf{1 0}$ could serve as the donor substrate to form high-affinity Siglec-15 ligands on the Lec2 cell surface, we then evaluated if these ligands could enforce interaction of modified cells with Siglec-15-expressing cells. Toward this end, we stained $\boldsymbol{\alpha 2 - 3 - 1 0}$ and $\boldsymbol{\alpha 2 - 6 - 1 0}$ displaying Lec2 $\mathrm{CHO}$ cells with CellTracker orange (CTO) as their identity marker. Unlabeled Lec2 CHO cells and cells labeled with the native ligand, a2-6-6, were used as controls. Subsequently, the Lec2 $\mathrm{CHO}$ cells stained with CTO were mixed with Siglec-15-expressing $\mathrm{CHO}$ cells that express extracellular Siglec-15 fused to intracellular GFP in a 100:1 ratio. The formation of cell clusters was determined by double staining and analyzed by flow cytometry and fluorescence microscopy (Fig. 5). Compared to the control non-sialylated Lec2 cells and cells modified with natural Neu5Ac, Lec2 cells modified with 10 induced significantly more cluster formation with Siglec15 -expressing cells.

While a2-6-10 was found to also bind to Siglec-2 weakly, osteoclasts and human osteoprogenitor cells (HOPs; $\mathrm{CD} 14^{+}$ peripheral blood mononuclear cells) do not express Siglec-2. Therefore, after confirming that cells displaying a2-6-10 could engage in cell-cell interactions via Siglec-15 binding, we assessed if Lec2 CHO cells displaying $\mathbf{\alpha 2 - 6 - 1 0}$ also could block osteoclast formation of RANKL -treated HOPs. RANKL, also known as osteoclast differentiation factor, is a cytokine secreted by osteoblasts that serves to activate osteoclasts, which are critically involved in bone resorption ${ }^{42}$. To determine if Lec2 $\mathrm{CHO}$ cells displaying a2-6-10 could block osteoclast formation from HOPs, HOPs were isolated and plated. Osteoclast differentiation was induced by the addition of human macrophage colonystimulating factor (hM-CSF) and RANKL ${ }^{46}$. Cells were allowed to differentiate for 7 days. On day 5, Lec2 $\mathrm{CHO}$ cells labeled with the high-affinity Siglec-15 glycan ligand, a2-6-10, or the native ligand, a2-6-6, were added to the plated cells. In these experiments, unlabeled Lec2 $\mathrm{CHO}$ cells were used as the negative control. On day 7, the formation of osteoclasts, which express abundant tartrate-resistant acid phosphatase (TRAP), was measured using the TRAP staining assay ${ }^{47}$. Osteoclasts were identified as TRAP-positive with 3 or more nuclei ${ }^{46,47}$. Remarkably, the addition of Lec2 $\mathrm{CHO}$ cells labeled with the high-affinity Siglec-15 ligand on day 5 resulted in over $50 \%$ reduction in osteoclast formation compared to the addition of unlabeled Lec2 $\mathrm{CHO}$ cells and HOPs treated with RANKEL and and hM-CSF only (Fig. 6). Furthermore, Lec2 $\mathrm{CHO}$ cells modified with a 2-6linked natural sialic acid did not exhibit significant effects on osteoclast formation presumably due to the relatively low affinity of $\boldsymbol{\alpha 2 - 6 - 6}$ for Siglec-15 compared to $\mathbf{\alpha 2 - 6 - 1 0}$ (Supplementary Fig. 4).

\section{Discussion}

The use of molecular diagnostic techniques for the detection of biological markers in the genome or proteome has dramatically increased over the last several decades, which has stemmed from the advancements in molecular biology techniques and the miniaturization of high-throughput microarrays ${ }^{48,49}$. Microarrays are widely used to study disease-specific gene mutations or protein expression and to correlate these expression signatures with disease progression. As a result, clinicians are increasingly able to treat patients according to their individual gene and/or protein expression profiles, known as personalized medicine. While microarrays comprised of nucleic acid or peptides/proteins are well-established and accessible for common users, glycan microarray technology remains highly specialized ${ }^{50}$.

The in-solution, cell-based glycan array platforms described here are developed to address this unmet need. By combining STs, ST6Gal1 and ST3Gal4, and a1-3FT with Lec2 $\mathrm{CHO}$ cells possessing a narrow and relatively homogeneous repertoire of $\mathrm{N}$ linked glycoforms, cell arrays displaying sialosides, fucosides and their structurally related analogs can be assembled easily using instruments available in most biology labs. With the necessary glycosyltransferases and nucleotide sugar donors prepared in advance, it only requires $2-3 \mathrm{~h}$ to fabricate a cell-based glycan array displaying $>30$ distinct glycan epitopes in a 96-well plate. By contrast, it takes days to weeks to assemble a conventional glycan array using comparable chemoenzymatic chemistry because each individual glycan to be printed needs to be synthesized and purified $^{4,7}$. Importantly, the diversity of cell-based glycan array platforms can be significantly expanded by including the recently developed CHO and HEK cell lines with simplified surface glycans via precision genome editing as foundation platforms ${ }^{51}$.

Using conventional glycan microarray technology, high-affinity and selective glycan ligands have been identified for six out of 15 human Siglecs, which involves the synthesis of hundreds of sialylated oligosaccharides bearing unnatural substituents ${ }^{35-38}$. The cell-based array technique described here has proven to be a simpler alternative for this endeavor. By using STs to install unnatural sialic acids directly onto the cell surface in a linkagespecific manner, the lengthy synthesis of sialylated oligosaccharides is avoided. Because sialylation reactions are performed on the cell surface, significantly fewer materials are required to produce the desired structures.

Recently, Boltje and co-workers reported an alternative method to discover Siglec ligands directly on the cell surface ${ }^{52,53}$. This method involved metabolic labeling using an alkyne functionalized ManNAc precursor followed by cellsurface CuAAC. Although a few high-affinity ligands were discovered by this approach, none were specific for a particular Siglec. This is likely due to the lack of linkage specificity of this

Fig. 4 Cell-based glycan arrays for screening Siglec-15 ligands. a The workflow for the construction of cell-based glycan arrays on the cell surface of Lec2 cells using a chemoenzymatic strategy for screening specific and high-affinity Siglec-15 ligands. Cells were first treated with ST6Gal1 and the alkyne functionalized CMP-sialic acid analog 7, followed by reacting with a library of azide-containing molecules via the biocompatible CuAAC to form a cellbased glycan array. The modified cells were incubated with Siglec-15-Fc pre-complexed with Anti-human IgG APC, then analyzed by flow cytometry. $\mathbf{b}$ Flow cytometry data presented in bar graph. Fold difference in MFI of Siglec-15 Fc binding was determined by comparison with the negative control (no transfer of 7) ( $n=3$, error bars are reported as SEM). c Structures of CMP-SiaA13 (10) and CMP-SiaA31 (11) and resulting glycan structures after sialyltransferase-mediated cell-surface glycosylation. d Siglec-15-Fc binding after the one-step labeling of Lec2 CHO cells using ST6Gal1, ST3Gal1 or ST3Gal4-mediated transfer of $\mathbf{1 0}$ or $\mathbf{1 1}$, respectively. Fold difference in MFI of Siglec-15 Fc binding was determined by comparison with the negative control $(n=3$, error bars are reported as SEM). e Evaluating the specificity of $\alpha 2-6$-linked Siglec-15 ligands, $\alpha \mathbf{2}-\mathbf{6 - 1 0}$ and $\alpha \mathbf{2 - 6}-\mathbf{- 1 1}$ ( $n=3$, error bars are reported as SEM). f Evaluating the specificity of $\alpha 2-3$-linked Siglec-15 ligands, $\alpha \mathbf{2 - 3 - 1 0}$ and $\alpha \mathbf{2 - 3 - 1 1}(n=3$, error bars are reported as SEM) 
approach. Most Siglecs differentially bind to sialosides based on their Neu5Ac linkage. Metabolic labeling leads to incorporation of unnatural sialic acids in both a2-3- and a2-6-linkages, largely complicating the downstream Siglec binding studies. By contrast, the cell-based microarray described here utilized STs to install unnatural sialic acids directly on cell-surface glycans linkage specifically.

Siglec-15 is constitutively expressed in osteoclasts. During the RANKL-induced osteoclast differentiation, Siglec-15 is considerably upregulated ${ }^{39-42}$. Several antibodies targeting RANKL
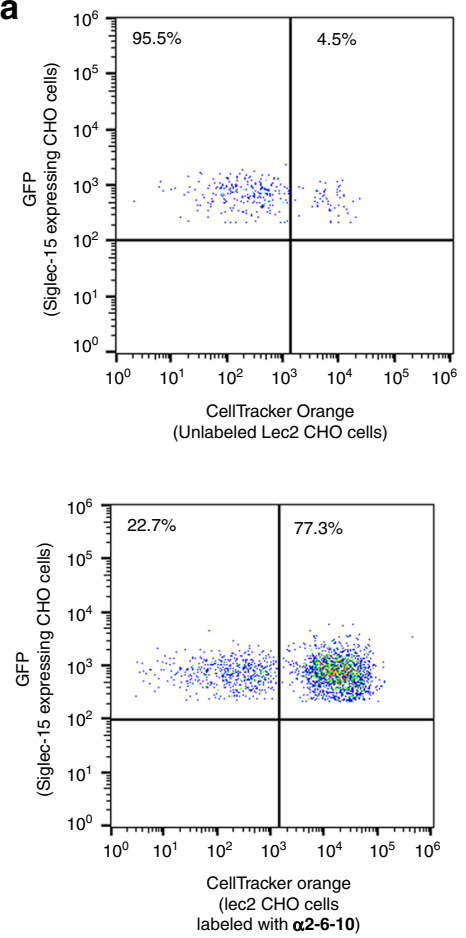

d

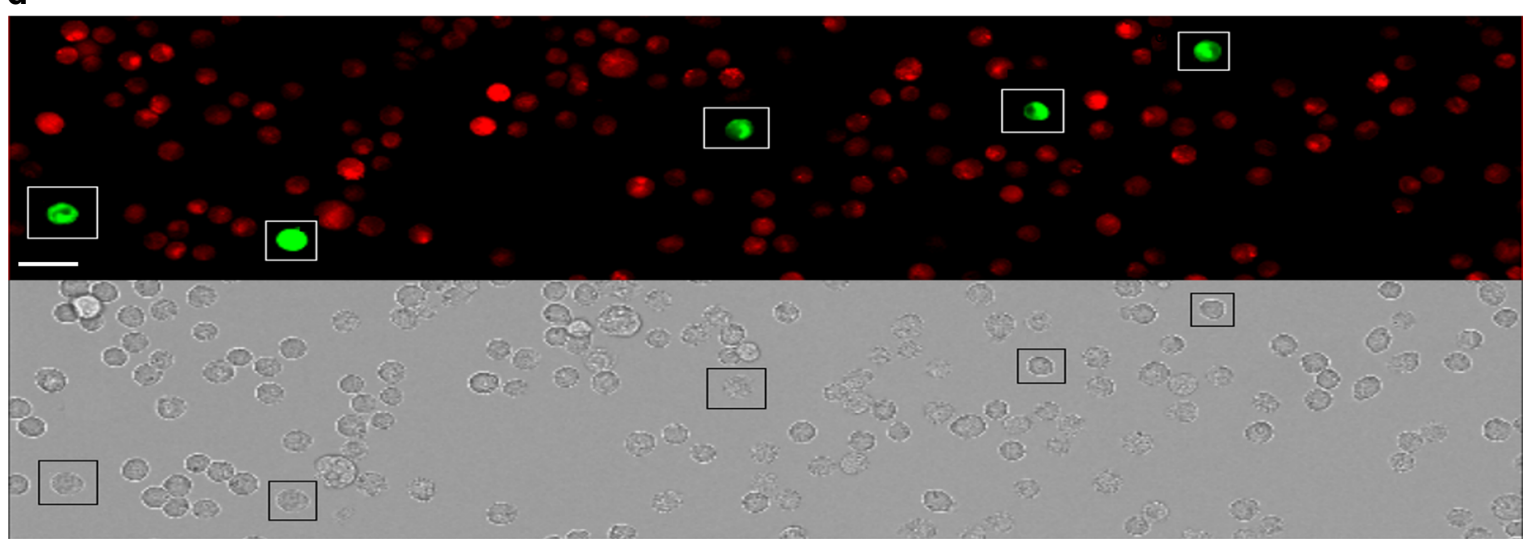

e

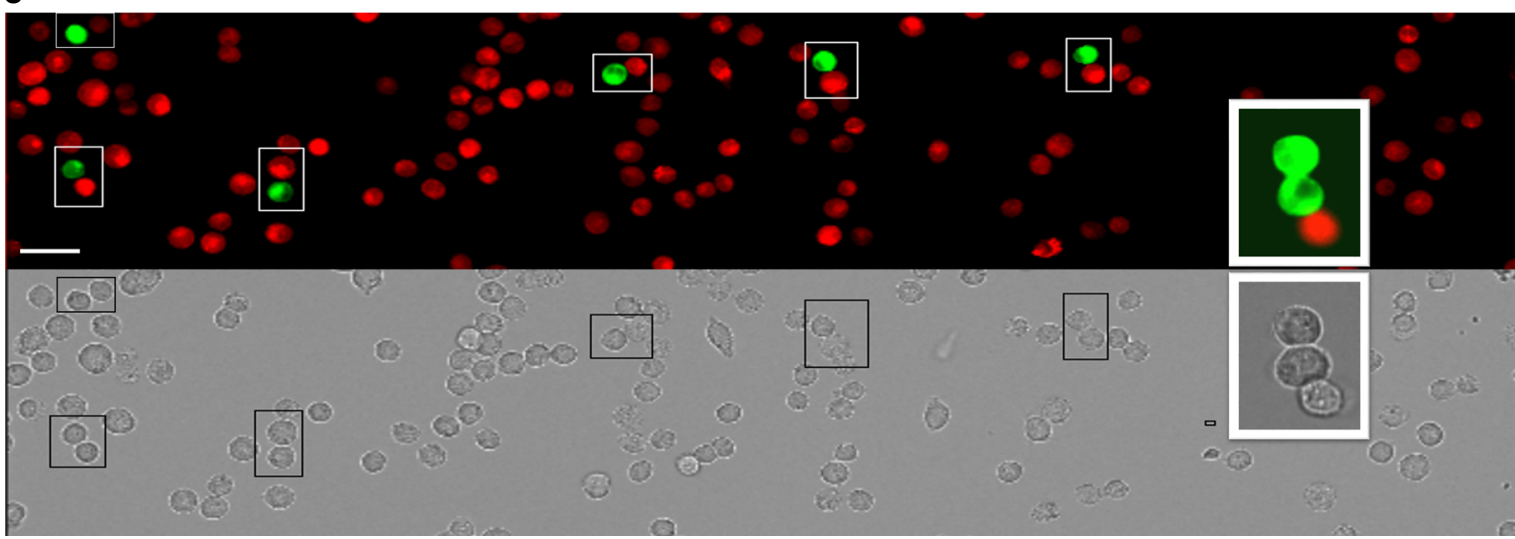

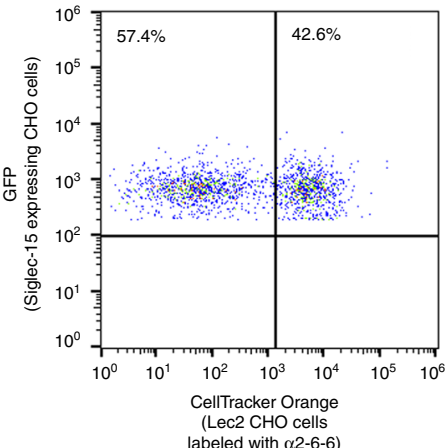
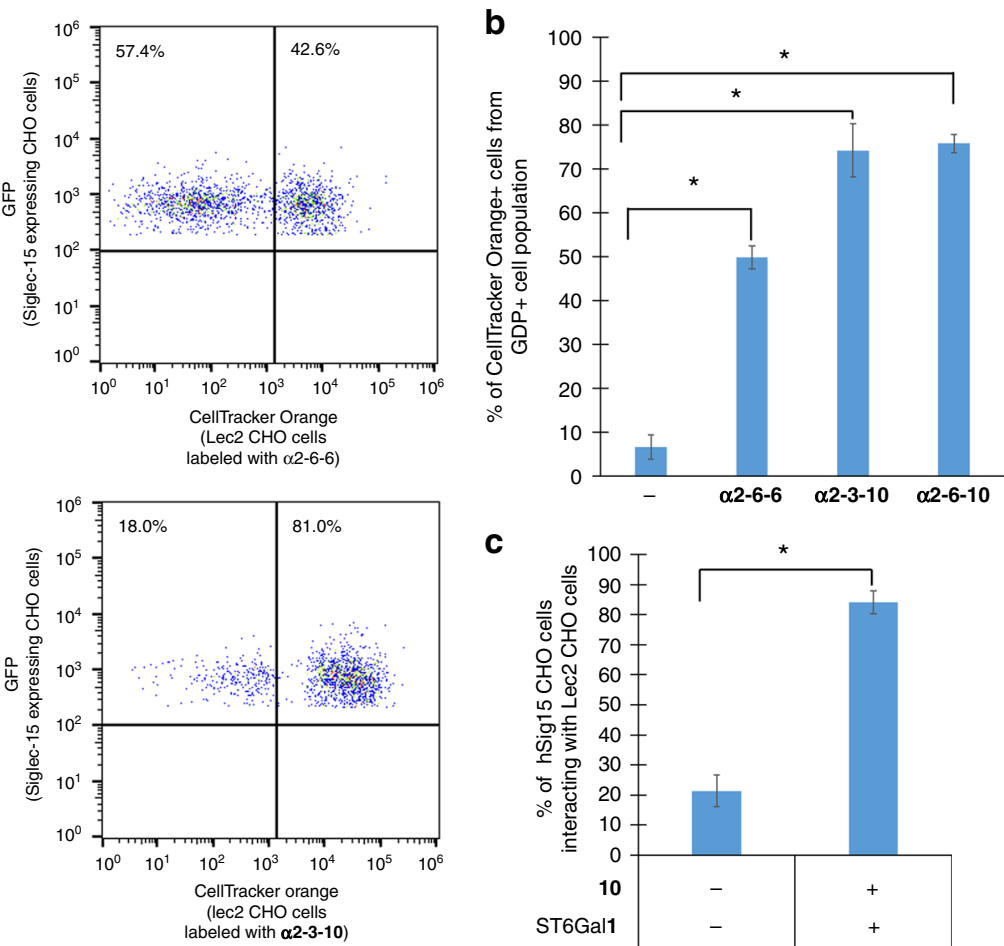

c

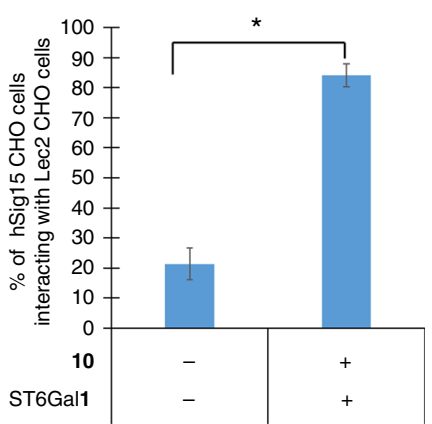


are FDA-approved, or are in late stage clinical trials for the treatment of osteoporosis and cancer-induced bone loss, and there are keen interests in targeting Siglec-15 for the same therapeutic applications ${ }^{54-56}$. In fact, Tremblay and co-workers discovered a monoclonal antibody for Siglec-15 that impaired osteoclast differentiation both in vitro and in vivo ${ }^{46}$. Exposure of human HOPs during differentiation to Lec2 $\mathrm{CHO}$ cells displaying the high-affinity Siglec-15 ligand (a2-6-10) impaired osteoclast formation, suggesting that this ligand may serve as the lead compound to be further optimized for clinical evaluation. While our method serves as a useful tool for the high-throughput screening of high-affinity glycan ligands for Siglecs and other GBPs, cells may display a range of glycans containing the modified monosaccharide. For the future evaluation of its therapeutic potential, isolation, purification, characterization, and validation of the high-affinity Siglec-15 ligand are required. Currently, we are in the process of using the cell-based glycan array technique to identify specific and high-affinity ligands for other Siglecs.

\section{Methods}

Cell culture conditions. Lec 2 and Lec 8 CHO cells were grown in monolayer in alpha-Minimum Essential medium ( $\alpha$-MEM) (Invitrogen) supplemented with 10\% fetal bovine serum (FBS) (Sigma-Aldrich). In all cases, cells were incubated in a $5.0 \%$ carbon dioxide, water-saturated incubator at $37^{\circ} \mathrm{C}$.

Synthesis of 10 and 11. CMP-SiaPoc (7) (10 mm) and azide A13 or A31 (12 mM) were dissolved in 1:1 DMSO/ $\mathrm{H}_{2} \mathrm{O}$ containing premixed 3-[4-(\{bis[(1-tert-butyl$1 \mathrm{H}-1,2,3$-triazol-4-yl)methyl]amino\}methyl)-1H-1,2,3-triazol-1-yl]propanol (BTTP)- $\mathrm{CuSO}_{4}$ complex $\left(2 \mathrm{~mm} \mathrm{CuSO}_{4}, 4 \mathrm{~mm} \mathrm{BTTP}\right)$ and $5 \mathrm{~mm}$ freshly prepared sodium ascorbate and agitated for $3 \mathrm{~h}$ at $30^{\circ} \mathrm{C}$. Reaction completion was monitored by TLC and LCMS. Upon consumption of 7 , the reaction was quenched with EDTA $(4 \mathrm{~mm})$. The mixture was concentrated in vacuo and crude reaction products were purified by Bio-Gel P2 gel filtration chromatography eluted with $\mathrm{NH}_{4} \mathrm{CO}_{3}(50 \mathrm{~mm})$. Only the fractions containing the product were collected and lyophilized.

General procedure of cell-surface in situ glycosylation. In situ fucosylation of cells was performed as previously described ${ }^{57}$. Briefly, cells were resuspended in $100 \mu \mathrm{L}$ HBSS buffer containing $20 \mathrm{~mm} \mathrm{MgSO}_{4}, 3 \mathrm{~mm}$ HEPES, $0.1 \%$ FBS, $50 \mu \mathrm{m}$ GDP-fucose analogs $(\mathbf{1}-\mathbf{5}), 30 \mathrm{mU}$ al-3FT. After incubation at $37^{\circ} \mathrm{C}$ for $10 \mathrm{~min}$, the cells were washed three times with PBS. In situ sialylated of cells was performed as previously described ${ }^{18,23}$. Briefly, cells were washed three times with PBS and resuspended in $100 \mu \mathrm{L}$ serum-free $\alpha$-MEM containing $0.65 \mu \mathrm{L}$ BSA $(2 \mathrm{mg} / \mathrm{mL})$, $0.65 \mu \mathrm{L}$ Shrimp Alkaline phosphatase (1000 U/mL, New England BioLabs), $31 \mu \mathrm{L}$ of $1.5 \mathrm{~m}$ sucrose, $4.2 \mu \mathrm{L} \mathrm{ST6Gall}{ }^{17}$ or ST $3 \mathrm{Gal} 4^{16}(1 \mathrm{mg} / \mathrm{mL})$ and $4 \mu \mathrm{L}$ CMP-sialic acid analogs $(6-11)(10 \mathrm{mM})$ with a density of $0.5-1.0 \times 10^{6} / 100 \mu \mathrm{L}$ in 96 -well plates at $37^{\circ} \mathrm{C}$ for $1 \mathrm{~h}$. The treated cells were then washed three times with PBS.

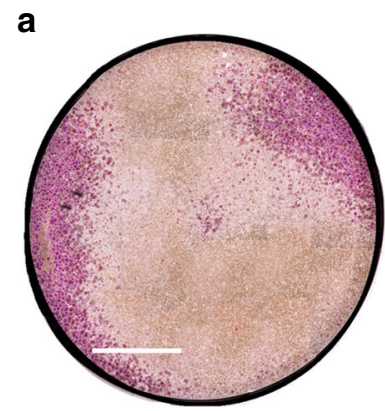

b

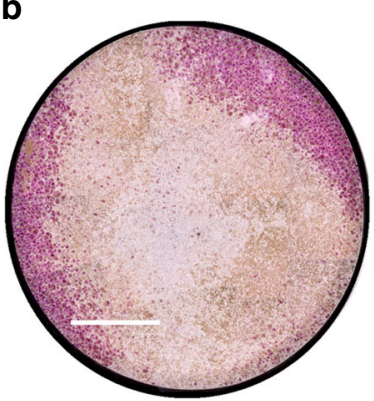

C

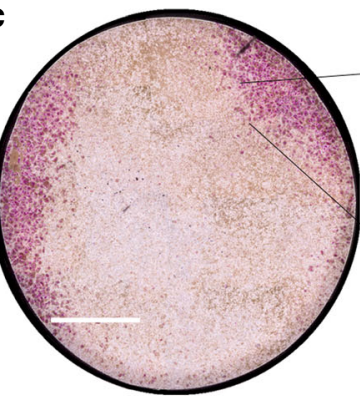

e

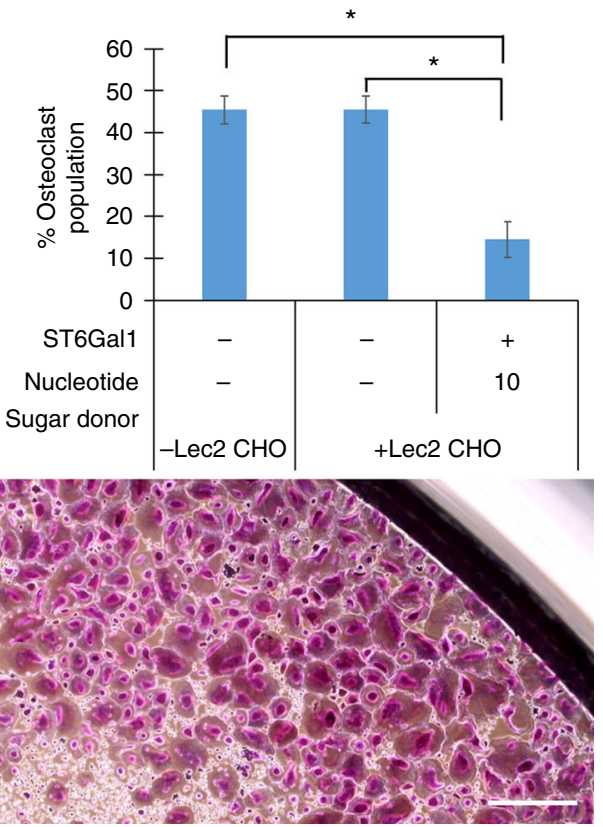

Fig. 6 Siglec-15 ligands impair osteoclast differentiation. Osteoclast differentiation was induced by the addition of hM-CSF and RANKL to the plated HOPs. On day 5, Lec2 CHO cells labeled with the high-affinity Siglec-15 glycan ligand, $\alpha \mathbf{2 - 6 - 1 0}$, or the native ligand, $\alpha \mathbf{2 - 6 - 6}$, were added to the plated cells. Quantitative analysis of osteoclast formation by a TRAP-staining assay was performed on day 7 on cells with no addition of Lec2 CHO cells (scale bar: 4 $\mathrm{mm}$ ) (a), with the addition of unlabeled Lec2 CHO cells (scale bar: $4 \mathrm{~mm}$ ) (b), and with the addition of Lec2 CHO cells labeled with $\alpha \mathbf{2 - 6 - 1 0}$ (scale bar: 4 $\mathrm{mm}$ ) (c). d Zoomed-in regions of osteoclast population (scale bar: $200 \mu \mathrm{m}$ ). e Percentage of well containing osteoclast populations (area of purple/entire well area $\times 100 \% ; n=3$, error bars are reported as SEM, significant difference compared with control was assessed using Student's $t$ test $\left({ }^{\star} P<0.01\right)$ )

Fig. 5 High-affinity Siglec-15 ligands bridge cell interactions. Lec2 CHO cells displaying high-affinity Siglec-15 ligands or unmodified Lec 2 CHO cells were stained with CTO and mixed with Siglec-15-expressing CHO cells that express extracellular Siglec-15 fused to GFP in the intracellular domain. The formation of cell clusters was determined by double staining and analyzed by flow cytometry and fluorescence microscopy. a Dot plot displaying GFP+ population (Siglec-15-expressing CHO cells) that are interacting with CTO-stained Lec2 CHO cells. b Percentage of GFP+ population (Siglec-15-expressing $\mathrm{CHO}$ cells) that interact with CTO-stained Lec2 $\mathrm{CHO}$ cells $(n=3$, error bars are reported as SEM, significant difference compared with control was assessed using Student's $t$-test $\left({ }^{\star} P<0.0001\right)$ ). c Percentage of Siglec-15-expressing $\mathrm{CHO}$ cells $(\mathrm{GFP}+)$ that interact with $\mathrm{CTO}$-stained Lec2 $\mathrm{CHO}$ cells by fluorescence microscopy $\left(n=3\right.$, error bars are reported as SEM, significant difference compared with control was assessed using Student's $t$-test ${ }^{\star} P<$ 0.0001)). d Fluorescence microscopy images of Siglec-15-expressing CHO cells (GFP+) and CTO-stained, unlabeled, Lec2 CHO cells (scale bar: $40 \mu \mathrm{m}$ ). e Fluorescence microscopy images of Siglec-15-expressing CHO cells (GFP+) interacting with CTO-stained Lec2 CHO cells labeled with $\alpha \mathbf{2 - 6 - 1 0}$ (scale bar: $40 \mu \mathrm{m})$ 
General procedure of cell-surface CuAAC. Alkyne-bearing cells, prepared as described above using the CMP-sialic acid analog 7 (CMP-SiaPoc), were reacted with $1 \mu \mathrm{L}$ azide ( $5 \mathrm{~mm}$ stock) in a $100 \mu \mathrm{L}$ PBS containing premixed BTTPS-CuSO ${ }_{4}$ complex $\left(75 \mu \mathrm{M} \mathrm{CuSO}_{4}, 450 \mu \mathrm{M}\right.$ BTTPS) and $2.5 \mathrm{~mm}$ freshly prepared sodium ascorbate for $5 \mathrm{~min}$. The $\mathrm{Cu}(\mathrm{I})$-catalyzed azide-alkyne cycloaddition was quenched by adding bathocuproine sulfonate (BCS, $1 \mathrm{~mm}$ )

Cell-surface lectins and antibody staining. Cells subjected to in situ fucosylation were assessed for binding of $\mathrm{Le}^{\mathrm{X}}$ lectins as follows. For SSEA-1, the cells were incubated with mouse monoclonal anti-SSEA-1 $(5 \mu \mathrm{g} / \mathrm{mL}, \mathrm{R} \& \mathrm{D}$ systems, Inc.) in $100 \mu \mathrm{L}$ PBS with $2 \%$ FBS for 30 min followed by staining with PE-conjugated antimouse-IgM $(5 \mu \mathrm{g} / \mathrm{mL}$, Jackson ImmunoResearch Laboratories, Inc.) in $100 \mu \mathrm{L}$ PBS with $2 \% \mathrm{FBS}$ for $40 \mathrm{~min}$. For AAL and Lotus A, the cells were incubated with Lotus A-FITC $(0.1 \mathrm{mg} / \mathrm{mL}$, EY Laboratories, Inc.), or AAL-FITC $(10 \mu \mathrm{g} / \mathrm{mL}$, Vector Laboratories) in $100 \mu \mathrm{L}$ PBS with $2 \%$ FBS for 40 min. Cells were then washed three times with PBS, and resuspended in PBS with 2\% FBS and 2 mM EDTA for flow cytometric analysis. Cells subjected to in situ sialylation were assessed for binding of LacNAc and sLacNAc lectins as follows. For ECA and SNA-I, the cells were incubated with ECA-FITC $(1 \mu \mathrm{g} / \mathrm{mL}$, EY Laboratories), or SNA-I-FITC $(1 \mu \mathrm{g} / \mathrm{mL}$, EY Laboratories) in $100 \mu \mathrm{L}$ PBS with $2 \%$ FBS for 30 min on ice. For MAL-II, the cells were incubated with MAL-II-FITC $(1 \mu \mathrm{g} / \mathrm{mL}$, EY Laboratories) in $100 \mu \mathrm{L}$ PBS with $2 \% \mathrm{FBS}$ and $1 \mathrm{mM} \mathrm{CaCl}_{2}$ for 30 min on ice. Cells were then washed three times with PBS, and resuspended in PBS with 2\% FBS and 2 mM EDTA for flow cytometric analysis.

Cell-surface binding of Siglec-Fc chimeras. Siglec-Fc chimeras containing the Nterminal Ig domains fused to the Fc region of human IgG1 were prepared as described previously ${ }^{35}$. Labeled Lec2 CHO cells were resuspended in $100 \mu \mathrm{L}$ of recombinant Siglec-Fc chimera supernatants pre-complexed with anti-human IgG APC (1:40 dilution, $200 \mu \mathrm{g} / \mathrm{mL})$. Briefly, $2.5 \mu \mathrm{L}$ of anti-Human IgG APC $(200 \mu \mathrm{g} /$ $\mathrm{mL}$ ) was added to $50 \mu \mathrm{L}$ of recombinant Siglec Fc chimera supernatant and incubated in the dark at room temperature for $15 \mathrm{~min}$ followed by dilution with 50 $\mu \mathrm{L}$ of PBS containing $1 \%$ BSA. Cells resuspended in this solution were incubated for $30 \mathrm{~min}$ on ice before being washed three times with PBS, and resuspended in PBS with $2 \%$ FBS and 2 mM EDTA for flow cytometric analysis.

Cloning and expression of Siglec-15 in $\mathbf{C H O}$ cells. Siglec-15 contains a lysine residue in its transmembrane region that pairs with Dap-12. Since Dap-12 is not expressed in standard cell lines (e.g. CHO), we took another approach to expressing the extracellular portion of human Siglec-15 as a transmembrane portion of Siglec- 15 by cloning a chimeric protein consisting of amino acids $1-260$ of human Siglec-15 with the transmembrane and cytoplasmic tail of human CD22 fused to a C-terminal eGFP. This chimeric protein was cloned into pcDNA5/FRT/TOP/V5/ His (Invitrogen) using the NheI and AgeI restriction enzymes. CHO Flp-in cells (Invitrogen) cells stably transfected with this vector according to the manufacturer's protocol. After selection of cells with Hygromycin-B for 2 weeks, all cells were found to be expressing high levels of GFP by flow cytometry.

Lec2 $\mathrm{CHO}$ cell and Siglec-15-expressing $\mathrm{CHO}$ cell clustering. For flow cytometry analysis, Lec2 $\mathrm{CHO}$ cells were labeled with CellTracker orange (CTO) following the manufacturer's protocols (ThermoFisher Scientific), sialylated (as described above) and mixed with hSig15-expressing $\mathrm{CHO}$ cells that also express GFP in a ratio of 100:1 for $20-30 \mathrm{~min}$ on ice prior to analysis by flow cytometry. The GFP + cell population was analyzed for CTO staining and interacting cells were defined as double stained. For fluorescence microscopy analysis, Lec2 $\mathrm{CHO}$ cells stained with CTO were sialylated as described above, mixed with hSig15expressing $\mathrm{CHO}$ cells that also express GFP in a ratio of 100:1 for $20-30 \mathrm{~min}$ on ice prior to plating and incubation at $37^{\circ} \mathrm{C}$ for $10 \mathrm{~min}$ to allow adherence. Plates were gently rinsed once with PBS and cell clustering was imaged by fluorescence microscopy. Images of each sample were analyzed for cell-cell interactions. The number of hSig $15 \mathrm{CHO}$ cells that were interacting with Lec2 $\mathrm{CHO}$ cells was counted and expressed as a percentage of the total number of hSig15 $\mathrm{CHO}$ cells analyzed.

Osteoclast differentiation. HOPs (CD14+ peripheral blood mononuclear cells) were isolated from normal human peripheral blood mononuclear cells using EasySep Human Monocyte Isolation Kit (StemCell Technologies) following the manufacturer's instructions. HOPs were plated at $0.5 \times 10^{6}$ cells/well in a 24 -well plate with $1 \mathrm{~mL}$ media (aMEM supplemented with $1 \mathrm{~mm}$ sodium pyruvate and $10 \%$ FBS). To stimulate osteoclast formation, $25 \mathrm{ng} / \mathrm{mL}$ of human macrophage colonystimulating factor and $30 \mathrm{ng} / \mathrm{mL}$ of human RANKL (R\&D Systems) were added. Cells were allowed to differentiate for 7 days with half media replaced every 3 days. On Day 5, Lec2 CHO cells $\left(0.1 \times 10^{6}\right.$ cells $)$ sialylated as described above using glycosyl donor 10 were added to wells. Unlabeled Lec2 CHO cells were used as a negative control. On Day 7, cells were fixed and permeablized followed by staining for TRAP using the TRAP kit following manufacturer protocols (Sigma Aldrich). Osteoclasts were defined as TRAP+ with 3 or more nuclei.
Data availability. All data are available from the authors upon reasonable request.

Received: 27 November 2017 Accepted: 30 January 2018

Published online: 28 February 2018

\section{References}

1. Haltiwanger, R. S. \& Lowe, J. B. Role of glycosylation in development. Annu. Rev. Biochem. 73, 491-537 (2004).

2. Rudd, P. M., Elliott, T., Cresswell, P., Wilson, I. A. \& Dwek, R. A. Glycosylation and the immune system. Science 291, 2370-2376 (2001).

3. Varki, A., Kannagi, R. \& Toole, B. P. In Essentials of Glycobiology 2nd edn (eds. Varki, A. et al.) 617-632 (Cold Spring Harbor Press, New York, 2009).

4. Feizi, T., Fazio, F., Chai, W. \& Wong, C. H. Carbohydrate microarrays-a new set of technologies at the frontiers of glycomics. Curr. Opin. Struct. Biol. 13, 637-645 (2003).

5. Oyelaran, O. \& Gildersleeve, J. C. Glycan arrays: recent advances and future challenges. Curr. Opin. Chem. Biol. 13, 406-413 (2009).

6. National Research Council. In Transforming Glycoscience: A Roadmap for the Future (ed. Walt, D.) 85-89 (The National Academies Press, Washington, DC, 2012).

7. Song, X., Heimburg-Molinaro, J., Cummings, R. D. \& Smith, D. F. Chemistry of natural glycan microarrays. Curr. Opin. Chem. Biol. 18, 70-77 (2014).

8. Yu, H. \& Chen, X. One-pot multienzyme (OPME) systems for chemoenzymatic synthesis of carbohydrates. Org. Biomol. Chem. 14, 2809-2818 (2016).

9. Wang, S. K. et al. Targeting the carbohydrates on HIV-1: Interaction of oligomannose dendrons with human monoclonal antibody 2G12 and DCSIGN. Proc. Natl. Acad. Sci. USA 105, 3690-3695 (2008).

10. Zhang, Y. \& Gildersleeve, J. C. General procedure for the synthesis of neoglycoproteins and immobilization on epoxide-modified glass slides. Methods Mol. Biol. 808, 155-165 (2012).

11. Huang, M. L. \& Godula, K. Nanoscale materials for probing the biological functions of the glycocalyx. Glycobiology 26, 797-803 (2016)

12. Patnaik, S. K. \& Stanley, P. Lectin-resistant $\mathrm{CHO}$ glycosylation mutants. Methods Enzymol. 416, 159-182 (2006).

13. Lopez Aguilar, A. et al. Tools for studying glycans: recent advances in chemoenzymatic glycan labeling. Acs Chem. Biol. 12, 611-621 (2017).

14. Soriano del Amo, D. et al. Chemoenzymatic synthesis of the sialyl Lewis X glycan and its derivatives. Carbohydr. Res. 345, 1107-1113 (2010).

15. Wang, W. et al. Chemoenzymatic synthesis of GDP-L-fucose and the Lewis X glycan derivatives. Proc. Natl. Acad. Sci. USA 106, 16096-16101 (2009).

16. Moremen, K. W. et al Expression system for structural and functional studies of human glycosylation enzymes. Nat. Chem. Biol. 14, 156-162 (2018).

17. Meng, L. et al. Enzymatic basis for N-glycan sialylation: structure of ratalpha2,6-sialyltransferase (ST6GAL1) reveals conserved and unique features for glycan sialylation. J. Biol. Chem. 288, 34680-34698 (2013)

18. Yu, S. H. et al. Selective exo-enzymatic labeling detects increased cell surface sialoglycoprotein expression upon megakaryocytic differentiation. J. Biol. Chem. 291, 3982-3989 (2016).

19. Oelmann, S., Stanley, P. \& Gerardy-Schahn, R. Point mutations identified in Lec8 Chinese hamster ovary glycosylation mutants that inactivate both the UDP-galactose and CMP-sialic acid transporters. J. Biol. Chem. 276, 26291-26300 (2001)

20. Cummings, R. D. \& Etzler, M. E. In Essentials of Glycobiology 2nd edn (eds. Varki, A. et al.) 633-648 (Cold Spring Harbor Press, New York, 2009).

21. Wu, A. M. et al. Differential affinities of Erythrina cristagalli lectin (ECL) toward monosaccharides and polyvalent mammalian structural units. Glycoconj. J. 24, 591-604 (2007).

22. Gross, H. J. \& Brossmer, R. Enzymatic transfer of sialic acids modified at C-5 employing four different sialyltransferases. Glycoconj. J. 12, 739-746 (1995).

23. Sun, T. et al. One-step selective exoenzymatic labeling (SEEL) strategy for the biotinylation and identification of glycoproteins of living cells. J. Am. Chem. Soc. 138, 11575-11582 (2016).

24. Crocker, P. R., Paulson, J. C. \& Varki, A. Siglecs and their roles in the immune system. Nat. Rev. Immunol. 7, 255-266 (2007).

25. Crocker, P. R. \& Varki, A. Siglecs, sialic acids and innate immunity. Trends Immunol. 22, 337-342 (2001).

26. Macauley, M. S., Crocker, P. R. \& Paulson, J. C. Siglec-mediated regulation of immune cell function in disease. Nat. Rev. Immunol. 14, 653-666 (2014).

27. Sullivan-Chang, L., O’Donnell, R. T. \& Tuscano, J. M. Targeting CD22 in Bcell malignancies: current status and clinical outlook. BioDrugs 27, 293-304 (2013).

28. Tanida, S. et al. Binding of the sialic acid-binding lectin, Siglec-9, to the membrane mucin, MUC1, induces recruitment of beta-catenin and subsequent cell growth. J. Biol. Chem. 288, 31842-31852 (2013). 
29. Laszlo, G. S., Estey, E. H. \& Walter, R. B. The past and future of CD33 as therapeutic target in acute myeloid leukemia. Blood Rev. 28, 143-153 (2014).

30. Chen, W. C. et al. In vivo targeting of B-cell lymphoma with glycan ligands of CD22. Blood 115, 4778-4786 (2010)

31. Chen, W. C. et al. Antigen delivery to macrophages using liposomal nanoparticles targeting sialoadhesin/CD169. PLoS ONE 7, e39039 (2012).

32. Kawasaki, N. et al. Targeted delivery of mycobacterial antigens to human dendritic cells via Siglec-7 induces robust T cell activation. J. Immunol. 193, 1560-1566 (2014).

33. Pegon, J. N. et al. Factor VIII and von Willebrand factor are ligands for the carbohydrate-receptor Siglec-5. Haematologica 97, 1855-1863 (2012).

34. Chen, W. C., Sigal, D. S., Saven, A. \& Paulson, J. C. Targeting B lymphoma with nanoparticles bearing glycan ligands of CD22. Leuk. Lymphoma 53, 208-210 (2012).

35. Rillahan, C. D., Schwartz, E., McBride, R., Fokin, V. V. \& Paulson, J. C. Click and pick: identification of sialoside analogues for Siglec-based cell targeting. Angew. Chem. Int. Ed. 51, 11014-11018 (2012).

36. Rillahan, C. D. et al. Disubstituted sialic acid ligands targeting Siglecs CD33 and CD22 associated with myeloid leukaemias and B cell lymphomas. Chem. Sci. 5, 2398-2406 (2014).

37. Blixt, O. et al. Sialoside analogue arrays for rapid identification of high affinity Siglec ligands. J. Am. Chem. Soc. 130, 6680-6681 (2008).

38. Rillahan, C. D. et al. On-chip synthesis and screening of a sialoside library yields a high affinity ligand for Siglec-7. Acs Chem. Biol. 8, 1417-1422 (2013).

39. Hiruma, Y., Hirai, T. \& Tsuda, E. Siglec-15, a member of the sialic acidbinding lectin, is a novel regulator for osteoclast differentiation. Biochem. Biophys. Res. Commun. 409, 424-429 (2011).

40. Hiruma, Y. et al. Impaired osteoclast differentiation and function and mild osteopetrosis development in Siglec-15-deficient mice. Bone 53, 87-93 (2013).

41. Ishida-Kitagawa, N. et al. Siglec-15 protein regulates formation of functional osteoclasts in concert with DNAX-activating protein of $12 \mathrm{kDa}$ (DAP12). J. Biol. Chem. 287, 17493-17502 (2012).

42. Kameda, Y. et al. Siglec-15 regulates osteoclast differentiation by modulating RANKL-induced phosphatidylinositol 3-kinase/Akt and Erk pathways in association with signaling Adaptor DAP12. J. Bone Miner. Res. 28, 2463-2475 (2013).

43. Angata, T., Tabuchi, Y., Nakamura, K. \& Nakamura, M. Siglec-15: an immune system Siglec conserved throughout vertebrate evolution. Glycobiology 17, 838-846 (2007).

44. Soriano del Amo, D. et al. Biocompatible copper(I) catalysts for in vivo imaging of glycans. J. Am. Chem. Soc. 132, 16893-16899 (2010).

45. Dall'Olio, F. The sialyl-alpha2,6-lactosaminyl-structure: biosynthesis and functional role. Glycoconj. J. 17, 669-676 (2000).

46. Stuible, M. et al. Mechanism and function of monoclonal antibodies targeting Siglec-15 for therapeutic inhibition of osteoclastic bone resorption. J. Biol. Chem. 289, 6498-6512 (2014).

47. Lv, Y. et al. Tartrate-resistant acid phosphatase $5 \mathrm{~b}$ is a marker of osteoclast number and volume in RAW 264.7 cells treated with receptor-activated nuclear kappaB ligand. Exp. Ther. Med. 9, 143-146 (2015).

48. Ashley, E. A. Towards precision medicine. Nat. Rev. Genet. 17, 507-522 (2016)

49. Pareek, C. S., Smoczynski, R. \& Tretyn, A. Sequencing technologies and genome sequencing. J. Appl. Genet. 52, 413-435 (2011).

50. Heimburg-Molinaro, J., Song, X., Smith, D. F. \& Cummings, R. D. Preparation and analysis of glycan microarrays. Curr. Protoc. Protein Sci. Chapter 12, https://doi.org/10.1002/0471140864.ps1210s64 (2011).

51. Yang, Z. et al. Engineered CHO cells for production of diverse, homogeneous glycoproteins. Nat. Biotechnol. 33, 842-844 (2015).
52. Bull, C. et al. Sialic acid glycoengineering using an unnatural sialic acid for the detection of sialoglycan biosynthesis defects and on-cell synthesis of Siglec ligands. Acs Chem. Biol. 10, 2353-2363 (2015).

53. Bull, C. et al. Steering Siglec-Sialic acid interactions on living cells using bioorthogonal chemistry. Angew. Chem. Int. Ed. 56, 3309-3313 (2017).

54. Miller, P. D. Denosumab: anti-RANKL antibody. Curr. Osteoporos. Rep. 7, 18-22 (2009).

55. Schwarz, E. M. \& Ritchlin, C. T. Clinical development of anti-RANKL therapy. Arthritis Res. Ther. 9(Suppl 1), S7 (2007).

56. Tanaka, R. \& Kumagai, Y. Pharmacokinetics of anti-RANKL antibody drugs: Denosumab. Clin. Calcium 26, 1597-1603 (2016)

57. Zheng, T. et al. Tracking $N$-acetyllactosamine on cell-surface glycans in vivo Angew. Chem. Int. Ed. 50, 4113-4118 (2011).

\section{Acknowledgements}

Financial supports from the NIH (GM093282 and GM113046 to P.W. and GM103390 to K.W.M.) and NSFC-Shandong Joint Fund for Marine Science Research Centers (U1606403) are gratefully acknowledged. We would like to thank Prof. J.C. Paulson for providing Siglec-Fc chimeras and for stimulating discussions, and Prof. Pamela Stanley for providing $\mathrm{CHO}$ Lec mutants.

\section{Author contributions}

P.W. conceived the concept of cell-based glycan array and supervised the project. M.S.M co-supervised the project and provided resources. J.G.B., H.J., K.W.M., M.S.M. and P.W. contributed to experimental design. K.W.M. provided reagents. J.G.B. and H.J. performed the experiments. J.G.B., M.S.M. and P.W. analyzed the data. J.G.B. wrote the manuscript, which was edited and approved by all authors.

\section{Additional information}

Supplementary Information accompanies this paper at https://doi.org/10.1038/s41467 018-03245-5.

Competing interests: The authors declare no competing financial interests.

Reprints and permission information is available online at http://npg.nature.com/ reprintsandpermissions/

Publisher's note: Springer Nature remains neutral with regard to jurisdictional claims in published maps and institutional affiliations.

cc (i) Open Access This article is licensed under a Creative Common Attribution 4.0 International License, which permits use, sharing, adaptation, distribution and reproduction in any medium or format, as long as you give appropriate credit to the original author(s) and the source, provide a link to the Creative Commons license, and indicate if changes were made. The images or other third party material in this article are included in the article's Creative Commons license, unless indicated otherwise in a credit line to the material. If material is not included in the article's Creative Commons license and your intended use is not permitted by statutory regulation or exceeds the permitted use, you will need to obtain permission directly from the copyright holder. To view a copy of this license, visit http://creativecommons.org/ licenses/by/4.0/.

(c) The Author(s) 2018 